\title{
Influência do lantânio nas propriedades estruturais do $\mathrm{SrSnO}_{3}$
}

\section{(The influence of lanthanum on the structural properties of $\mathrm{SrSnO}_{3}$ )}

\author{
M. R. Cassia-Santos ${ }^{1 *}$, S. B. Mendes ${ }^{l}$, M. F. C. Gurgel ${ }^{1}$, A. T. Figueiredo ${ }^{1}$, \\ M. Godinho Jr. ${ }^{1}$, C. E. M. Braz ${ }^{2}$, E. Longo ${ }^{2}$ \\ ${ }^{1} D Q$, Universidade Federal de Goiás - CAC, Av. Dr. Lamartine Pinto de Avelar 1120, Setor Universitário, \\ Catalão, GO 75704-020 \\ ${ }^{2} I Q$, Universidade Estadual Paulista - UNESP, R. Prof. Francisco Degni 55, Bairro Quitandinha, Araraquara, \\ SP 14800-060 \\ *mrcsantos@gmail.com
}

\begin{abstract}
Resumo
Os estanatos com estrutura perovskita têm apresentado propriedades promissoras à sua utilização como cerâmica eletrônica do tipo: sensores, catalisadores e mais recentemente, fotoluminescente. Neste trabalho foi analisado o estudo do efeito do lantânio nas propriedades estruturais do estanato de estrôncio e a avaliação como sistema fotoluminescente. Os pós de $\mathrm{La}_{\mathrm{x}} \mathrm{Sr}_{1-\mathrm{x}} \mathrm{SnO}_{3}$ foram sintetizados pelo método do precursor polimérico e tratados termicamente a 600,800 e $1000{ }^{\circ} \mathrm{C}$ por 4 h. Os sistemas foram caracterizados por análise térmica, difração de raios $\mathrm{X}$, análise de área de superfície específica, espectroscopia no UV-Visível, no infravermelho, microscopia eletrônica de varredura e medidas fotoluminescentes. A partir dos resultados de difração de raios $\mathrm{X}$ observa-se a formação de um sistema cristalino monofásico com estrutura perovskita cúbica, tanto em função da temperatura de calcinação como para as adições de lantânio (1, 5 e 10\% em mol). Apesar da adição de lantânio no $\mathrm{SrSnO}_{3}$, a estrutura perovskita se mantém estável. O tamanho de cristalito diminui com a adição de lantânio, o que indica que o lantânio pode estar agindo como possível inibidor de crescimento de partícula e, conseqüentemente, diminuindo o tamanho de cristalito. Os resultados de área de superfície especifica mostram que a adição de lantânio favorece, de forma geral, a formação de sólidos com maior área. Os valores de "gap" óptico obtidos pela teoria de Tauc estão entre 3,81 e 3,98 eV, apresentando um aumento gradativo em função da adição de lantânio. Os pós tratados a $600^{\circ} \mathrm{C}$ com $1 \%$ em mol de lantânio são os que apresentam maior intensidade de emissão fotoluminescente. Palavra-chave: estanatos, perovskita, lantânio, fotoluminescência.
\end{abstract}

\begin{abstract}
The tin compounds with perovskite structure have shown promising properties for use as electronic ceramics such as sensors, catalysts and most recently, photoluminescent materials. In this work the effect of lanthanum on the structural properties of La S $r_{l}$. $\mathrm{SnO}_{3}$ and the evaluation of this system as photoluminescent material were studied. The $\mathrm{La} \mathrm{S}_{x} \mathrm{r}_{1-x} \mathrm{SnO}_{3}$ powders were synthesized by the polymeric precursor method and thermal treatments at 600, 800 and $1000{ }^{\circ} \mathrm{C}$ for $4 \mathrm{~h}$. The systems were characterized by thermal analysis, X-ray diffraction, specific surface area measurements, UV-Visible spectroscopy, infrared spectroscopy, scanning electron microscopy and photoluminescence measurements. X-ray diffraction analysis shows crystalline phase with cubic perovskite structure, as a function of calcination temperature for the additions of lanthanum (1, 5 and 10 mol\%). Despite the addition of lanthanum in $\mathrm{SrSnO}_{3}$, the perovskite structure is stable. The crystallite size decreased with the addition of lanthanum, indicating that this dopant may be acting as a possible particle growth inhibitor and hence decreasing crystallite size. The results of the specific surface area show that the addition of lanthanum favors, in general, the formation of higher surface area solids. Values of the optical gap, according to the theory of Tauc, are between 3.81 and $3.98 \mathrm{eV}$, showing a gradual increase due to the addition of lanthanum. The powders doped with $1 \mathrm{~mol} \%$ of lanthanum and treated at $600{ }^{\circ} \mathrm{C}$ presented higher photoluminescent emission intensity.
\end{abstract}

Keywords: stannates, perovskite, lanthanum, photoluminescence.

\section{INTRODUÇ̃̃OO}

Os sistemas cerâmicos constituem uma classe de materiais economicamente viáveis e, a cada dia que passa, são cada vez mais utilizados mundialmente. $\mathrm{O}$ grande diferencial desses materiais está na potencialização de suas propriedades físicas e químicas que refletem direta e indiretamente em suas aplicações em diversas áreas. As diferentes propriedades destes materiais com diferentes estruturas têm atraído os pesquisadores da área de desenvolvimento de novos materiais por apresentarem, especialmente quando ligados a metais alcalinos, características de grande potencial para aplicações no campo da eletrônica [1], como em capacitores termicamente estáveis, sensores para uma variedade de gases, como o $\mathrm{CO}$ e $\mathrm{NO}_{\mathrm{x}}$, catalisadores, materiais para degradação fotocatalítica. Investigações sistemáticas têm sido realizadas, dando ênfase na síntese destes materiais, na relação de formação de fase, fornecendo as suas características estruturais e morfológicas, apresentando algumas das características elétricas dos corpos sintetizados, tais como os sensores [2,3]. 
Estanatos de alcalinos terrosos com fórmula geral $\mathrm{MSnO}_{3}(\mathrm{M}=\mathrm{Ca}, \mathrm{Sr}$ e Ba$)$ tem apresentado, recentemente, propriedades muito interessantes com grande potencial para aplicação na área de cerâmica eletrônica, tais como: capacitores, termicamente estáveis com baixa permissividade, sensores e catalisadores. De forma interessante, a literatura atual mostra que o comportamento $\mathrm{MgSnO}_{3}$ e do $\mathrm{Mg}_{2} \mathrm{SnO}_{4}$ é totalmente diferente dos estanatos de $\mathrm{Ca}, \mathrm{Sr}$ e $\mathrm{Ba}$ correspondentes e que o método de síntese, assim como a adição de dopantes, é um dos principais fatores na definição das propriedades finais destes sistemas [2-4].

Óxidos com estrutura tipo perovskita de fórmula geral $\mathrm{ABO}_{3}$, apresentam muitas propriedades importantes e podem ser utilizados em diferentes aplicações [2]. Dentre os óxidos com estrutura perovskita com grande aplicabilidade, o estanato de estrôncio tem apresentado excelentes resultados como um material dielétrico de importância tecnológica. O estanato do estrôncio, $\mathrm{SrSnO}_{3}$, já foi relatado como um dos materiais que podem ser usados nos sensores de umidade [3]. As soluções sólidas de titanatos e de estanatos de alcalinos terrosos são usadas também para a fabricação de capacitores cerâmicos. Alguns autores mostram que $\mathrm{SrSnO}_{3}$ é um material dielétrico de importância tecnológica, normalmente sintetizado acima de $1000{ }^{\circ} \mathrm{C}$ pela reação do estado sólido entre $\mathrm{SrCO}_{3}$ ou $\mathrm{SrO}$ e $\mathrm{SnO}_{2}$, cristalizando-se no sistema cúbico de uma perovskita. Este óxido tem se destacado por suas propriedades dielétricas, e recentemente por suas propriedades fotoluminescentes e fotocatalíticas [4]. Os estanatos de alcalinos-terrosos normalmente têm apresentado estrutura perovskita, entre eles $\mathrm{BaSnO}_{3}$, $\mathrm{CaSnO}_{3}$ e $\mathrm{SrSnO}_{3}$ [5]. A estrutura perovskita apesar de já ter sido estudada durante muitos anos está relacionada com as propriedades dos materiais. A substituição dos sítios A/B da estrutura perovskita pode ocasionar mudanças nas propriedades do produto final. A partir da adição de dopantes podem-se adquirir novas propriedades dos materiais ou mesmo intensificação de uma propriedade já existente. $\mathrm{O}$ $\mathrm{SrSnO}_{3}$ também tem se destacado por suas aplicações em função de dopantes como: $\mathrm{Ba}^{2+}, \mathrm{Ca}^{2+}, \mathrm{Ni}^{2+}, \mathrm{Nd}^{3+} \mathrm{e} \mathrm{La}^{3+}[2$, $3,6,7]$.

Neste contexto, o objetivo do estudo consiste em avaliar a influência da adição de lantânio nas propriedades estruturais, ópticas e morfológicas do $\mathrm{SrSnO}_{3}$ utilizando o método do precursor polimérico.

\section{EXPERIMENTAL}

Foram utilizados os reagentes: nitrato de lantânio hexahidratado P.A., hidróxido de amônio, etilenoglicol P.A., cloreto de estanho II dihidratado, carbonato de estrôncio, ácido nítrico, e ácido cítrico anidro. As massas dos reagentes utilizados na síntese dos compostos à base de $\mathrm{La}_{x} \mathrm{Sr}_{1-\mathrm{x}} \mathrm{SnO}_{3}$ foram calculadas estequiometricamente, sendo: $\mathrm{x}=1,5$ e $10 \%$ em mol. A obtenção dos estanatos ocorre inicialmente pela síntese do citrato de estanho a partir do $\mathrm{SnCl}_{2}$, o qual, sob agitação e aquecimento constante o ácido cítrico é dissolvido em água destilada. A esta solução aquosa adiciona-se o cloreto de estanho. A relação entre os íons cloreto de estanho e o ácido cítrico foi 1:3. Com uma bureta, foi adicionado o hidróxido de amônio à solução de cloreto de estanho e ácido cítrico. Após a formação de um precipitado branco, a eliminação do cloreto foi realizada com sucessivas lavagens. Para confirmar a eliminação de cloreto da solução, foi utilizada uma solução de nitrato de prata $(1 \mathrm{~mol} / \mathrm{L})$. O citrato de estanho obtido foi então secado em estufa por $12 \mathrm{~h}$ em no máximo $80^{\circ} \mathrm{C}$. Em seguida, realiza-se a obtenção do citrato de estanho com a adição de carbonato de estrôncio à solução de citrato de estanho com ácido nítrico e síntese da resina polimérica com a subseqüente adição de etilenoglicol (promoção da polimerização), que ocorre sob aquecimento e agitação a $80{ }^{\circ} \mathrm{C}$ por $2 \mathrm{~h}$. Na síntese do citrato de estanho/estrôncio foi utilizada a razão molar 3:1 de ácido cítrico:metal. Para a síntese da resina o etilenoglicol foi adicionado na solução de ácido cítrico/metal na proporção mássica de 40:60. Após a obtenção das resinas poliméricas realizou-se a primeira etapa de calcinação a $300{ }^{\circ} \mathrm{C}$ por horas para obtenção do pó precursor, que foi macerado e homogeneizado em peneiras de 200 mesh e tratado termicamente a 600,800 e $1000{ }^{\circ} \mathrm{C}$ por $4 \mathrm{~h}$ para obtenção dos óxidos de interesse.

A síntese de $\mathrm{La}_{x} \mathrm{Sr}_{1-\mathrm{x}} \mathrm{SnO}_{3}$ é realizada da mesma forma que o puro, acrescida da etapa de adição de lantânio, que ocorre após a obtenção do citrato de estanho/estrôncio com adição do nitrato de lantânio, sob agitação em média de $1 \mathrm{~h}$. Faz-se a calcinação para obtenção do pó precursor e, posteriormente, o tratamento térmico de 600 a $1000{ }^{\circ} \mathrm{C}$ por $4 \mathrm{~h}$. Em seguida à obtenção dos pós, foram realizadas as caracterizações.

A caracterização da estrutura cristalina dos pós de $\mathrm{La}_{\mathrm{x}} \mathrm{Sr}_{1-\mathrm{x}} \mathrm{SnO}_{3}$ foi feita por difração de raios $\mathrm{X}$ em um difratômetro XRD-6000 Shimadzu com radiação Cuka $(\lambda=1,54148 \mathrm{~nm})$, no intervalo angular na faixa $10^{\circ} \leq 2 \theta \leq$ $50^{\circ}$, passo para contagem de $0,02^{\circ}$ e o tempo de contagem de $3 \mathrm{~s}$ por passo. Como padrão de difração foi utilizado o silício cristalino. Os espectros de absorção na região do infravermelho foram obtidos em amostras prensadas com $\mathrm{KBr}$ em espectrofotômetro IRPrestige 21 Shimadzu. Os espectros de UV-VIS também foram obtidos para cálculo do "gap" óptico das amostras e o equipamento utilizado foi Cary 50 Varian, no modo refletância, registrados à temperatura ambiente na região entre 190 e $1000 \mathrm{~nm}$. As análises de área de superfície específica foram realizadas num aparelho ASAP-2420 pela técnica de adsorção gasosa com nitrogênio. Para análise da forma dos pós foi utilizado um microscópico eletrônico de varredura de alta resolução Zeiss FEG Supra 35. As medidas de emissão fotoluminescente foram feitas com comprimento de onda de excitação $350,7 \mathrm{~nm}$ obtido de um laser com íons de kriptônio (Coherent Innova), com uma potência de saída do laser de $200 \mathrm{~mW}$. O monocromador utilizado foi um Thermal Jarrel-Ash Monospec 27 e uma fotomultiplicadora Hamamatsu R446 acoplada a um sistema de aquisição composto de um "lock-in" SR-530 controlado por um microcomputador. 


\section{RESULTADOS E DISCUSSÃO}

\section{Análise térmica}

Os materiais com diferentes concentrações de lantânio foram analisados por análise térmica diferencial com a finalidade de avaliar a decomposição térmica. As curvas de TG e DTG para o $\mathrm{SrSnO}_{3}$ são mostradas na Fig. 1. Nesta figura são observados na TG/DTG processos de perda de massa em relação à eliminação de matéria orgânica original

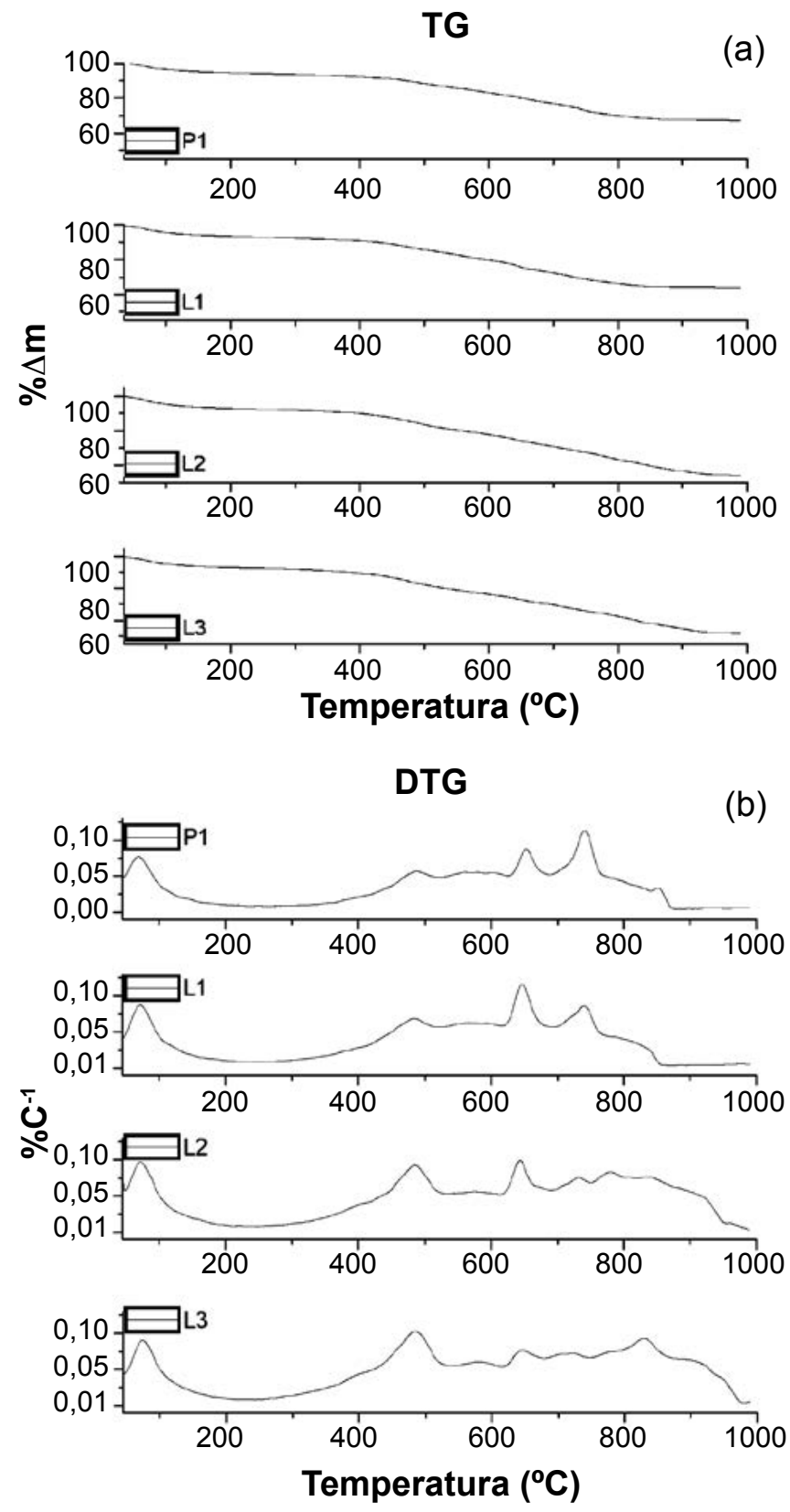

Figura 1: Curvas de TG (a) e DTG (b) para os pós de $\mathrm{La}_{\mathrm{x}} \mathrm{Sr}_{1-\mathrm{x}} \mathrm{SnO}_{3}$, em função da concentração em porcentagem molar de lantânio, calcinadas a $300{ }^{\circ} \mathrm{C} / 2 \mathrm{~h}$ : P1 (0\% de La), L1 (1\% La), L2 (5\% La), L3 (10\%).

[Figure 1: TG (a) and DTG (b) curves of $\mathrm{La}_{x} \mathrm{Sr}_{1-x} \mathrm{SnO}_{3}$ powders as a function of molar concentration of lanthanum calcined at $300{ }^{\circ} \mathrm{C}$ for 2 h: P1 (0\% La), L1 (1\% La) , L2 (5\% La), L3 (10\%).] do processo em $\mathrm{CO}_{2}$ e $\mathrm{H}_{2} \mathrm{O}$. A análise de DTA para a amostra de $\mathrm{SrSnO}_{3}$ apresenta o processo de pirólise (eliminação da matéria orgânica em $\mathrm{CO}_{2}$ e $\mathrm{H}_{2} \mathrm{O}$ ) além da perda de $\mathrm{H}_{2} \mathrm{O}$ e gases adsorvidos na superfície do pó [2]. No presente estudo, a desidratação do material e eliminação de gases adsorvidos na superfície é verificada com um pico endotérmico em $\sim 90{ }^{\circ} \mathrm{C}$. Em $884{ }^{\circ} \mathrm{C}$ observa-se um pico endotérmico referente a uma transição de fase, a qual será avaliada pelos resultados de difração de raios X.

\section{Difração de raios $X$}

Na Fig. 2 são apresentados os difratogramas dos pós de $\mathrm{SrSnO}_{3}$ em função da temperatura de tratamento térmico. Os padrões de difração para estes pós indicam a formação um sistema cristalino monofásico com estrutura perovskita cúbica em todas as temperaturas de calcinação em estudo. A $600{ }^{\circ} \mathrm{C}$ observa-se, pelo difratograma, a obtenção de um pó com baixo grau de cristalinidade, ainda amorfo, no entanto com picos de difração característicos da estrutura perovskita. A identificação da estrutura cristalina e da fase referente ao $\mathrm{SrSnO}_{3}$ foi realizada comparando os dados experimentais com o arquivo JCPDS 22-1442.

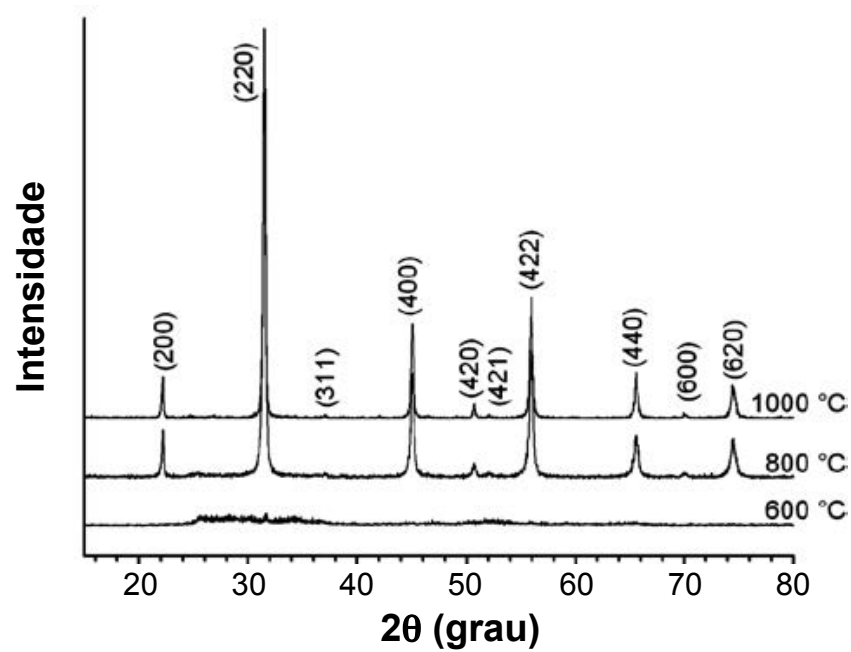

Figura 2: Difratogramas de raios $\mathrm{X}$ para pós de $\mathrm{SrSnO}_{3}$ calcinados em diferentes temperaturas por $4 \mathrm{~h}: 600,800$ e $1000^{\circ} \mathrm{C}$.

[Figure 2: X-ray diffraction patterns of the $\mathrm{SrSnO}_{3}$ powders calcined at different temperatures for $4 \mathrm{~h}: 600,800$ and $1000^{\circ} \mathrm{C}$.]

Na Fig. 3 são ilustrados os resultados de difração obtidos para as amostras de $\mathrm{La}_{\mathrm{x}} \mathrm{Sr}_{1-\mathrm{x}} \mathrm{SnO}_{3}$ calcinadas a $1000^{\circ} \mathrm{C} / 4 \mathrm{~h}$, na qual se observa que os pós possuem maior grau de cristalinidade em função do aumento da temperatura de calcinação. Por estes resultados têm-se que a adição de lantânio, em até $10 \%$ em mol, não promoveu modificações visíveis na rede do $\mathrm{SrSnO}_{3}$, dentro do limite de deteç̧ão do equipamento, observando-se apenas a formação da perovskita. Pode-se afirmar que a adição de lantânio não resultou na formação de fase secundária formando assim, solução sólida com o estanato de estrôncio. Como o raio iônico dos componentes

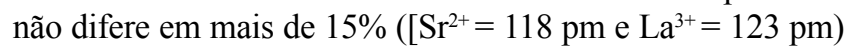


$[1,8]$, o lantânio pode entrar no sítio A da estrutura cristalina como substituinte do estrôncio.

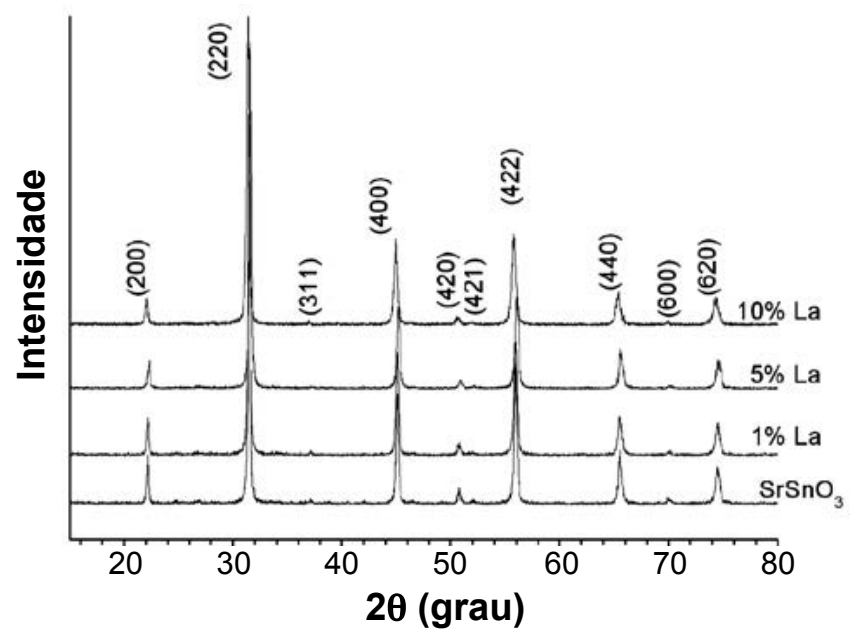

Figura 3: Difratogramas de raios $\mathrm{X}$ dos pós de $\mathrm{SrSnO}_{3}$ com diferentes concentrações de lantânio, calcinados a $1000^{\circ} \mathrm{C} / 4 \mathrm{~h}$.

[Figure 3: X-ray diffraction patterns of the $\mathrm{SrSnO}_{3}$ powders with different lanthanum concentration, calcined at $1000^{\circ} \mathrm{C} / 4 \mathrm{~h}$.]

$\mathrm{O}$ tamanho médio de cristalitos (TC) e a largura-à-meiaaltura (FWHM), em função da concentração de dopantes, foram calculados pelos dados do pico de difração de $100 \%$ de intensidade, plano (2 0 0), utilizando a equação de Scherrer (Tabela I). TC decresce com o aumento da concentração de lantânio, o que deve estar correlacionado com a migração de $\mathrm{La}^{3+}$ para a superfície ou regiões de contorno das partículas dificultando o seu crescimento, o que indica que o dopante age como um possível inibidor de crescimento de partícula $[1,9]$. Os valores de FWHM aumentam, relativamente, com o aumento da concentração de lantânio evidenciando o aumento do grau de desordem em função da entrada de dopante na rede cristalina do estanato.

Tabela I - Valores de TC e FWHM para pós de $\mathrm{La}_{\mathrm{x}} \mathrm{Sr}_{1-\mathrm{x}} \mathrm{SnO}_{3}$, calcinados a $1000{ }^{\circ} \mathrm{C} / 4 \mathrm{~h}$, em função da concentração de lantânio.

[Table I - TC and FWHM value of $\mathrm{La} \mathrm{Sr}_{1-x} \mathrm{SnO}_{3}$ powders, calcined at $1000{ }^{\circ} \mathrm{C} / 4 \mathrm{~h}$, as a function of lanthanum concentration.]

\begin{tabular}{cccc}
\hline \% lantânio & FWHM & TC $(\mathrm{nm})$ & $\begin{array}{c}\text { Parâmetro de } \\
\text { rede }(\mathrm{nm})\end{array}$ \\
\hline 0 & 0,208 & 198,694 & 8,055 \\
1 & 0,214 & 195,277 & 8,044 \\
5 & 0,241 & 182,141 & 8,044 \\
10 & 0,272 & 169,611 & 8,059 \\
\hline
\end{tabular}

\section{Analisador de área superficial (BET)}

Observa-se dos dados de área superficial específica (BET) valores de $5,194 \mathrm{~m}^{2} / \mathrm{g}$ para $\mathrm{SrSnO}_{3}$ antes da adição
Tabela II - Valores de área superficial específica para pós de $\mathrm{La}_{\mathrm{x}} \mathrm{Sr}_{1-\mathrm{x}} \mathrm{SnO}_{3}$ em função da concentração de lantânio e temperatura de calcinação.

[Table II - Specific surface area values for $\mathrm{La}_{x} \mathrm{Sr}_{1-x} \mathrm{SnO}_{3}$ powders, as a function of lanthanum concentration and calcination temperature.]

\begin{tabular}{ccccc}
\hline & \multicolumn{5}{c}{$\begin{array}{c}\text { Área Superficial Específica }\left(\mathrm{m}^{2} / \mathrm{g}\right), \\
\text { em função da \% em mol de lantânio. } \\
\left({ }^{\circ} \mathrm{C}\right)\end{array}$} & \multicolumn{4}{c}{ eratura } & \multicolumn{3}{c}{ em } & 5 & 10 \\
\hline 600 & 9,645 & 6,348 & 11,251 & 5,404 \\
800 & 19,896 & 28,374 & 15,255 & 8,660 \\
1000 & 5,194 & 9,347 & 10,238 & 7,745 \\
\hline
\end{tabular}

de lantânio. Após a adição de 1, 5 e $10 \%$ em mol de lantânio este valor passa para 9,347, 10,238 e 7,745 m²/g, respectivamente (Tabela II), resultados estes para amostras calcinadas a $1000{ }^{\circ} \mathrm{C} / 4 \mathrm{~h}$. A adição de lantânio, de forma geral, aumenta a área superficial do estanato de estrôncio, levando à formação de partículas menores. Tal resultado está em concordância com os resultados de TC, no qual se observa o decréscimo do TC com o aumento da concentração de lantânio. Esse resultado evidencia o efeito inibidor do lantânio no crescimento das partículas. Alguns autores mostram que dopantes de valência +3 , como o lantânio, migram para regiões de contorno dificultando os processos de transporte de massa e por conseqüência inibindo o crescimento de partícula levando a formação de pós com maior área superficial [10-12].

\section{Espectros vibracionais de absorção no infravermelho}

Vibrações moleculares são pequenas distorções da geometria das moléculas. A excitação dessas moléculas por radiação infravermelha é a base da espectroscopia IV. A ocorrência da radiação infravermelha pode ocorrer quando através dessa vibração ocorre uma mudança no momento dipolar elétrico de uma molécula [8]. Os espectros de infravermelho das amostras de $\mathrm{La}_{\mathrm{x}} \mathrm{Sr}_{1-\mathrm{x}} \mathrm{SnO}_{3}$ são apresentados na Fig. 4.

A vibração de alongamento $\mathrm{Sn}-\mathrm{O}$ está localizada em $535 \mathrm{~cm}^{-1}$ [5] e no presente estudo podem ser observadas de $520-535 \mathrm{~cm}^{-1}$. O grupo estanato apresenta vibrações na região espectral entre $600-700 \mathrm{~cm}^{-1}$ e entre $300-400 \mathrm{~cm}^{-1}$. A primeira foi observada nesse trabalho na faixa de 660$669 \mathrm{~cm}^{-1}$. No espectro do $\mathrm{BaSnO}_{3}$ picos em 700, 800 e 1050 podem ser atribuídos a vestígios de carbonato; apesar disso o carbonato pode ser considerado como uma impureza sem qualquer influencia na estrutura do composto. As bandas de absorção atribuídas às vibrações do carbonato estão localizadas na região entre 400 e $1800 \mathrm{~cm}^{-1}$. Pode-se ressaltar que as intensidades destas bandas referentes ao grupo carbonato diminuem com o aumento da temperatura de calcinação [13]. Geralmente a decomposição completa do carbonato ocorre a temperaturas superiores a $1473 \mathrm{~K}$ [14]. As principais bandas características do carbonato de bário são em 870 e $1430 \mathrm{~cm}^{-1}$, e neste trabalho foram encontradas 


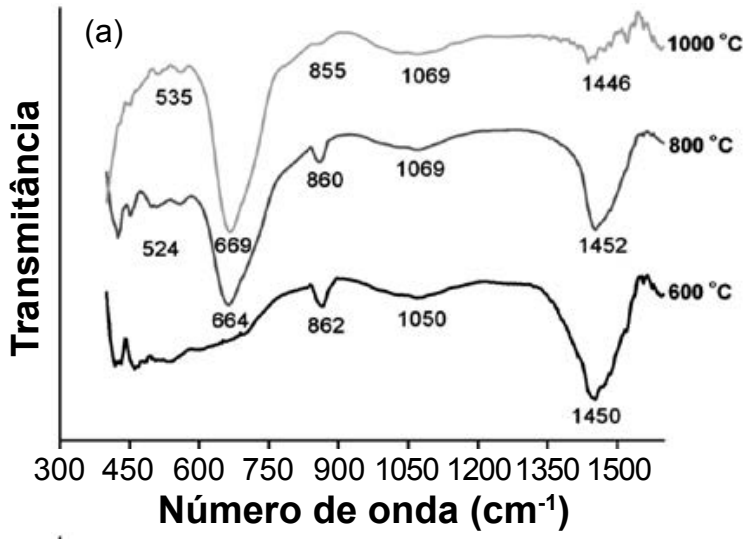

(c)

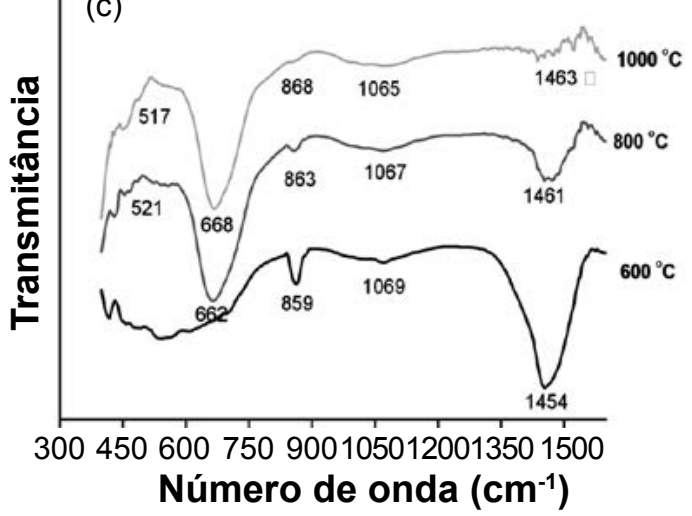

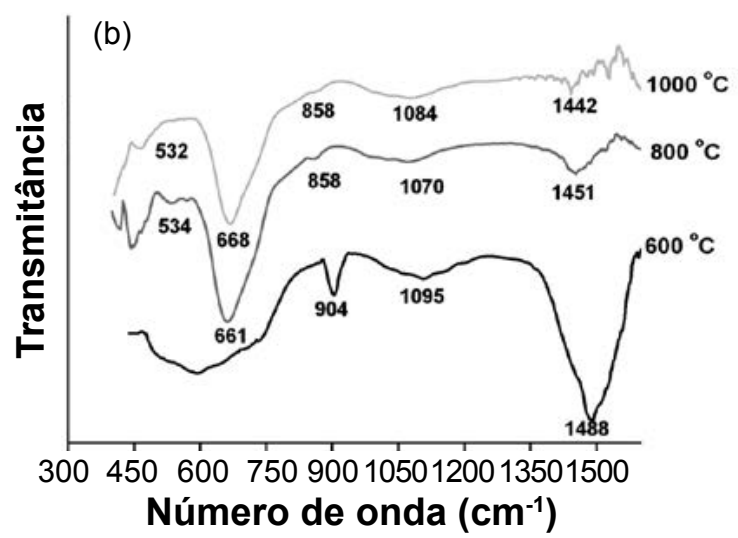

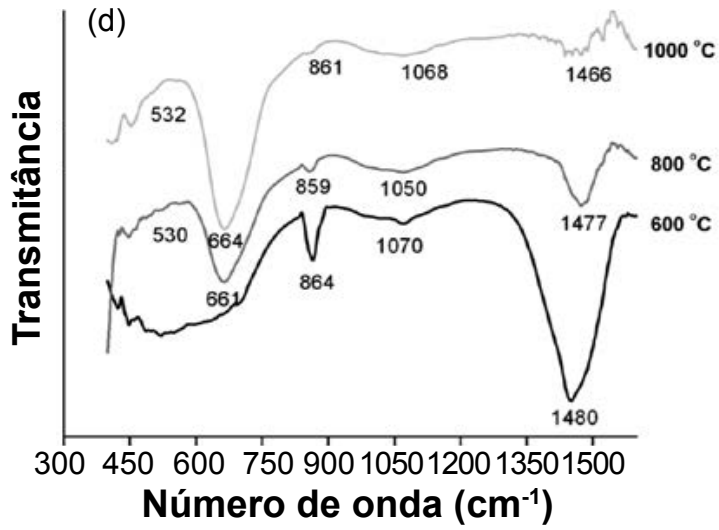

Figura 4: Espectros de infravermelho de pós de $\mathrm{La}_{\mathrm{x}} \mathrm{Sr}_{1-\mathrm{x}} \mathrm{SnO}_{3}$ calcinados em diferentes temperaturas $\left(600,800 \mathrm{e} 1000{ }^{\circ} \mathrm{C} / 4 \mathrm{~h}\right)$ para $\mathrm{x}$ : (a) 0; (b) 1 ; (c) 5 e (d) $10 \%$ em mol.

[Figure 4: Infrared spectra of $\mathrm{La}_{x} \mathrm{Sr}_{1-x} \mathrm{SnO}{ }_{3}$ powders, calcined at different temperatures (600, 800 and $1000{ }^{\circ} \mathrm{C} / 4$ h) for $x$ : (a) 0 ; (b) 1 ; (c) 5 and (d) $10 \mathrm{~mol} \%$.]

em $860-890 \mathrm{~cm}^{-1}$ e em $1445-1480 \mathrm{~cm}^{-1}$. Os pós de $\mathrm{SrSnO}_{3}$ após a adição de lantânio apresentam os mesmos modos vibracionais do grupo estanato, não sofrendo alterações significativas.

\section{Emissão fotoluminescente}

As análises luminescentes foram realizadas nas amostras de $\mathrm{SrSnO}_{3}$ puro e com adição de lantânio, tratadas termicamente a 300 e $600{ }^{\circ} \mathrm{C}$ por $4 \mathrm{~h}$. O material tratado a $600{ }^{\circ} \mathrm{C}$ apresenta a maior intensidade de emissão, tanto para as amostras de $\mathrm{SrSnO}_{3}$ puro quanto para materiais dopados. Esta propriedade está associada à desordem estrutural e térmica dos sistemas. Com o aumento da temperatura de calcinação, a ordem estrutural começa a aumentar a médio e longo alcance, logo as transições elétron-buraco sofrem uma diminuição levando ao decréscimo da intensidade do sinal fotoluminescente [15]. Existe um consenso geral de que a desordem estrutural é responsável pela emissão FL à temperatura ambiente em materiais, de tal modo que um material totalmente ordenado não apresenta emissão FL. Também, um material totalmente desordenado não apresenta emissão FL. Sendo assim, uma mínima ordem em um sistema desordenado é necessária para o material exibir FL.

A amostra de $\mathrm{SrSnO}_{3}$ apresenta emissão fotoluminescente mesmo sem adição de lantânio; no entanto, a adição de apenas 1\% em mol de lantânio promove um aumento significativo na intensidade fotoluminescente a 300 e $600{ }^{\circ} \mathrm{C}$. A análise dos espectros dos pós puros e com adição de lantânio (calcinados a 300 e $600{ }^{\circ} \mathrm{C} / 4 \mathrm{~h}$ ) mostra que os pós calcinados a $600{ }^{\circ} \mathrm{C}$ apresentam maior emissão luminescente do que os pós calcinados a $300{ }^{\circ} \mathrm{C}$. A $300{ }^{\circ} \mathrm{C}$ os pós contendo 0,1 e $5 \%$ em mol de lantânio apresentam uma relação de ordemdesordem muito próxima e, portanto, não apresentam variação significativa na emissão fotoluminescente. A $300{ }^{\circ} \mathrm{C}$ todas as amostras apresentaram emissão fotoluminescente com intensidade máxima em aproximadamente $635 \mathrm{~nm}$. Os pós calcinados a $600{ }^{\circ} \mathrm{C}$ apresentaram maior emissão do que os pós calcinados a $300{ }^{\circ} \mathrm{C}$, Fig. 5b. O resultado observado para as amostras contendo $1 \%$ de lantânio está atribuído à relação de ordem-desordem em função da concentração de defeitos estruturais e térmicos ideais para promover uma altaemissão. A $600{ }^{\circ} \mathrm{C}$, o pó puro e com adição de $1 \%$ em mol de lantânio, apresentaram maior intensidade de emissão fotoluminescente na região de $565-575 \mathrm{~nm}$ e as amostras com 5 e $10 \%$ em mol em 615 nm. Este resultado pode ser atribuído ao aumento da concentração de dopante e imperfeições em função da presença de lantânio proporcionando mudanças no grau de ordem-desordem dos sistemas. O sistema com 10\% em mol de lantânio apresenta evidências de recombinação de defeitos no sentido de diminuir o grau de desordem, aumento na ordem do sistema, levando ao decréscimo da emissão fotoluminescente. As amostras obtidas pelo método do precursor polimérico 
quando calcinado a $300{ }^{\circ} \mathrm{C}$ apresentam uma grande quantidade de matéria orgânica além da fase inorgânica do estanato. Uma vez que a emissão fotoluminescente, FL, é devida à fase inorgânica desordenada, à medida que diminui o carbono pelo aumento da temperatura de queima em atmosfera oxidante, a emissão FL aumenta. No entanto, vários são os trabalhos que apresentam resultados mostrando que este grau de desordem apresenta um limite, ou seja, à medida que se aumenta a temperatura de calcinação e/ou a concentração de impurezas, responsáveis pela desordem estrutural, observa-se um aumento do grau de cristalinidade do sistema e o decréscimo da FL [15, 19-21]. A diminuição da intensidade fotoluminescente para o sistema contendo $10 \%$ em mol de lantânio pode estar associada ao possível ordenamento do material, tal como discutido para filmes de $\mathrm{PbTiO}_{3}$ dopados com lantânio [16]. Os autores observaram que as propriedades fotoluminescentes destes materiais são
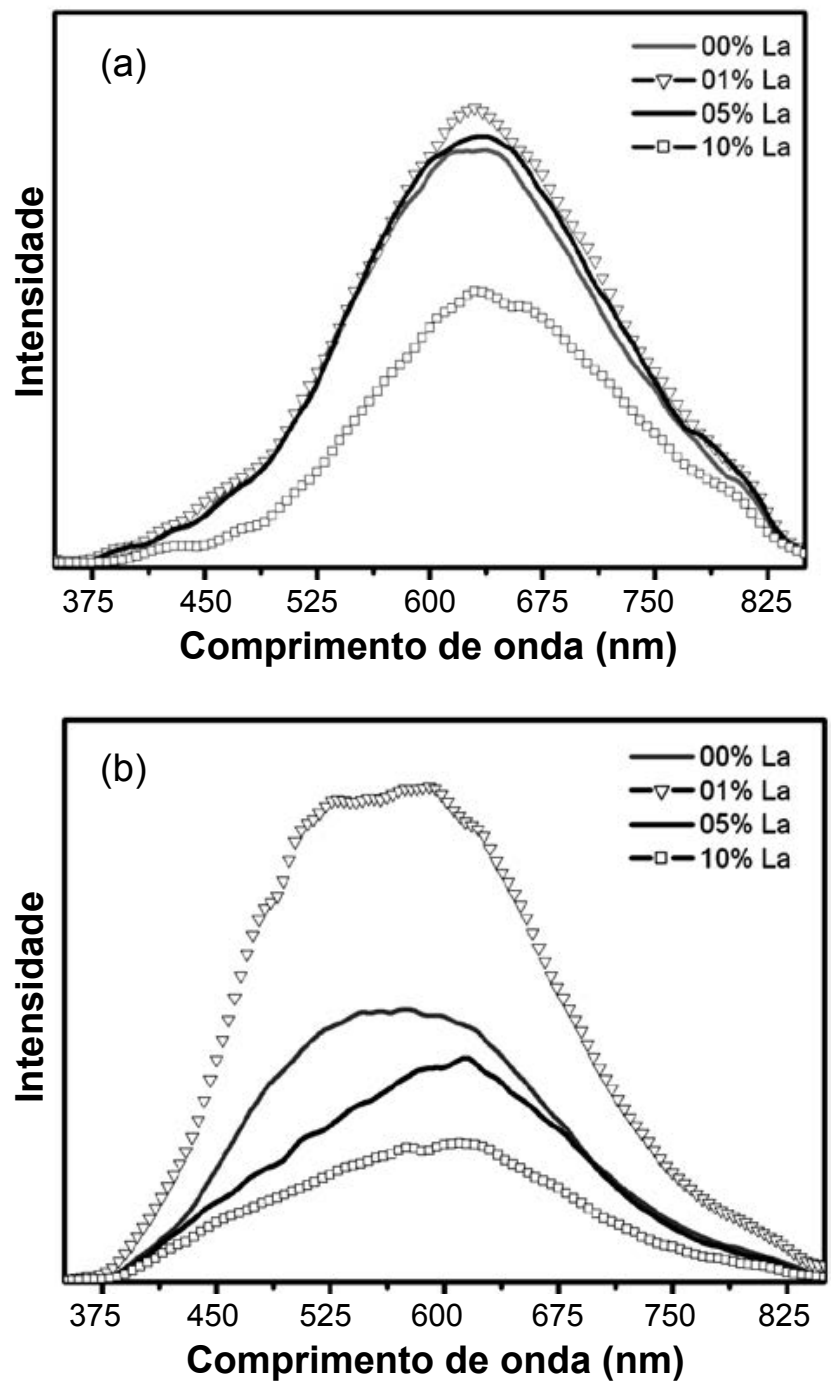

Figura 5: Espectros de fotoluminescência para $\mathrm{SrSnO}_{3}$ puro e com adição de lantânio em função da temperatura de calcinação: (a) 300 e (b) $600{ }^{\circ} \mathrm{C}$.

[Figure 5: PL spectroscopy of $\mathrm{SrSnO}_{3}$ pure and with lanthanum addition in function of the temperature of calcined: (a) 300 and (b) $\left.600^{\circ} \mathrm{C}.\right]$
Tabela III - "Gap" óptico dos pós de $\mathrm{La}_{\mathrm{X}} \mathrm{Sr}_{1-\mathrm{X}} \mathrm{SnO}_{3}$ em função da concentração de lantânio e da temperatura de calcinação. [Table III - Optical gap of $\mathrm{La}_{x} \mathrm{Sr}_{1-x} \mathrm{SnO}_{3}$ powders as a function of lanthanum concentration and calcination temperature.]

\begin{tabular}{ccccc}
\hline Temperatura & \multicolumn{4}{c}{ "gap" (eV) para } \\
$\left({ }^{\circ} \mathrm{C}\right)$ & 0 & 1 & 5 & 10 \\
\hline 600 & 3,70 & 3,66 & 3,65 & 3,75 \\
800 & 3,80 & 3,74 & 3,73 & 3,83 \\
1000 & 3,83 & 3,90 & 3,97 & 3,98 \\
\hline
\end{tabular}

fortemente alteradas com altas concentrações de lantânio $(20 \%$ em mol). O comprimento de onda correspondente à máxima intensidade FL normalizada também é influenciado pela concentração do dopante. Isto sugere que a substituição do chumbo pelo lantânio promove um aumento no "gap" do material. Um início de cristalização diminui a desordem do material eliminando os defeitos e, consequentemente, provoca um decréscimo na emissão fotoluminescente.

Na Tabela III são apresentados os valores de "gap" dos pós de $\mathrm{SrSnO}_{3}$ puro e com adição de lantânio determinados a partir das curvas de absorbâncias em função do comprimento de onda (método de Tauc [17]), em que se observa sempre um valor de "gap" menor para o sistema que ainda possui alto grau de desordem, $600^{\circ} \mathrm{C}$ (material ainda amorfo), devido à existênciade estados eletrônicos na região entre a banda de valência e a banda de condução. Estudos teóricos mostraram significativas diferenças entre as densidades eletrônicas, do estado desordenado e ordenado para o $\mathrm{CaTiO}_{3}$, que foram diretamente relacionadas ao fenômeno de fotoluminescência observado neste material [15].

Dos resultados apresentados tem-se que o aumento da temperatura de calcinação leva a uma maior organização do sistema e promove um aumento nos valores de "gap" de todos os sistemas em estudo Os resultados de espectroscopia na região do UV-Visível permitem avaliar a influência do dopante nos processos de transição eletrônica, sendo assim, adição do elemento dopante ocasionou um pequeno acréscimo nos valores do "gap". Os pós apresentam valores de "gap" próximos aos demostrados pela literatura no valor de 4,1 eV [18]. O "gap" óptico é controlado pelo grau de desordem, estrutural e térmico, na rede do composto, bem como a absorção está associada aos novos estados localizados no "gap". Sendo assim, observa-se uma maior intensidade de emissão FL para o material que está mais ordenado, porém ainda com um grau de desordem estrutural, Fig. 2 e Tabela I, ou seja, com apenas 1\% em mol de lantânio aumenta-se a concentração dos buracos-elétrons em função da formação de defeitos promovendo a emissão FL.

\section{Microscopia eletrônica}

Na Fig. 6 são apresentadas as fotomicrografias obtidas por microscopia eletrônica de varredura de alta resolução para as amostras de $\mathrm{La}_{\mathrm{X}} \mathrm{Sr}_{1-\mathrm{X}} \mathrm{SnO}_{3}$ calcinadas a $1000{ }^{\circ} \mathrm{C} / 4 \mathrm{~h}$. 

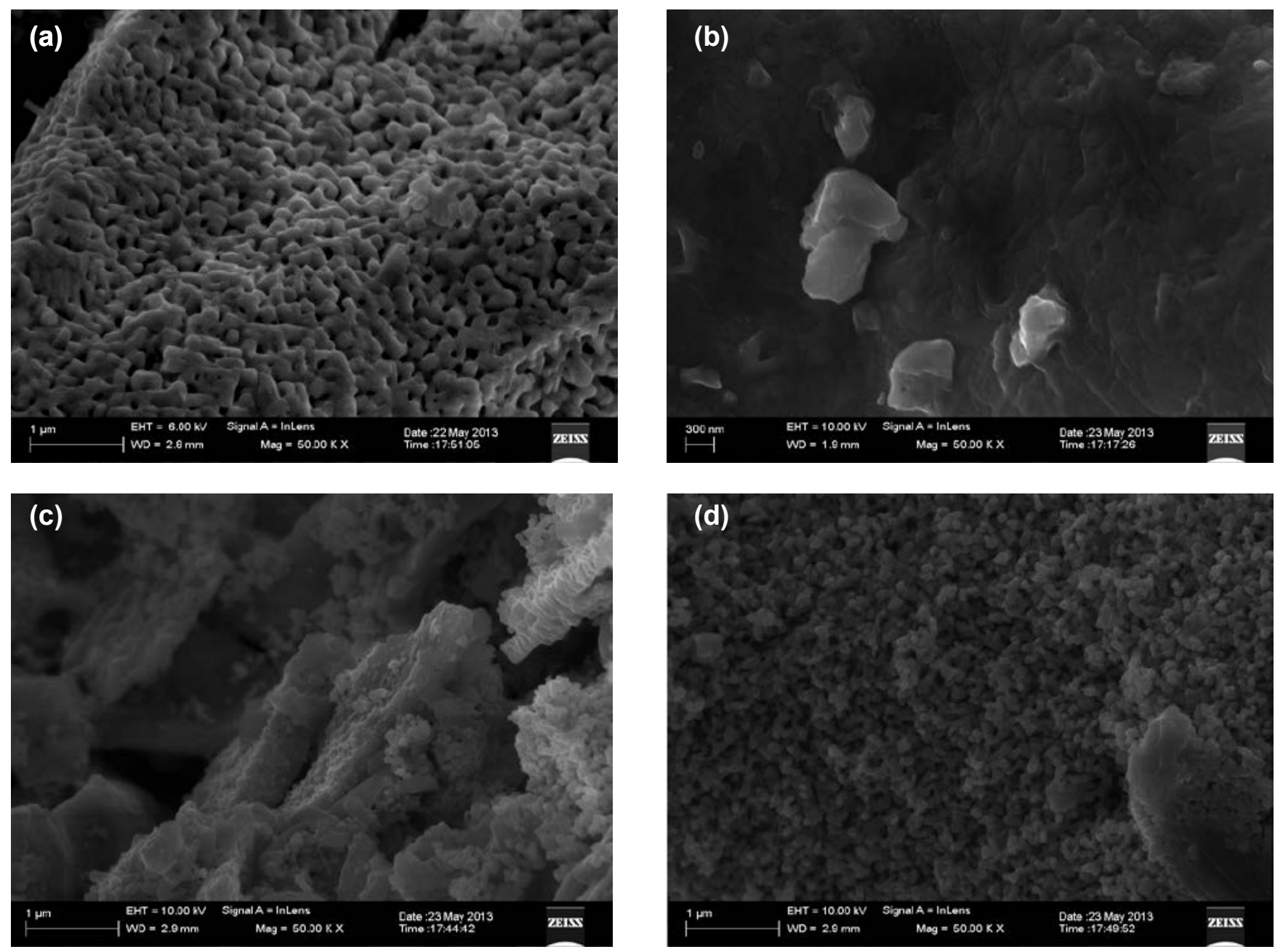

Figura 6: Fotomicrografias obtidas por microscopia eletrônica de varredura de alta resolução dos pós de $\mathrm{SrSnO}_{3}$ calcinados a $1000{ }^{\circ} \mathrm{C} / 4$ h: (a) puro, (b) com $1 \%$, (c) $5 \%$ e (d) $10 \%$ em mol de lantânio.

[Figure 6: FEG-SEM micrographs of $\mathrm{SrSnO}{ }_{3}$ powders calcined at $1000{ }^{\circ} \mathrm{C} / 4 \mathrm{~h}:$ (a) undoped, (b) doped with $1 \%$, (c) $5 \%$ and (d) $10 \%$ in mol of lanthanum.]

Observa-se destas microestruturas que a adição de lantânio à matriz de $\mathrm{SrSnO}_{3}$ promove o decréscimo do tamanho de partícula, tal como discutido anteriormente. $\mathrm{Na}$ amostra de $\mathrm{SrSnO}_{3}$ tem-se o início do processo de sinterização das partículas, com morfologia esférica e tamanho médio de partículas em torno de 120-150 nm. A adição de 5 e 10\% em mol de lantânio promove a formação de partículas mais esféricas e com um tamanho médio de partículas de $80 \mathrm{~nm}$. Da adição de 1\% em mol de lantânio observa-se uma maior aglomeração das partículas e o início da formação de uma fase lamelar.

\section{CONCLUSÕES}

Uma solução sólida de $\mathrm{La}_{\mathrm{x}} \mathrm{Sr}_{1-\mathrm{x}} \mathrm{SnO}_{3}$ foi sintetizada pelo método do precursor polimérico. A adição de lantânio promoveu a obtenção de partículas nanométricas com estrutura perovskita cúbica, que com o aumento da concentração de defeitos estruturais e térmicos tem-se uma significativa contribuição para as propriedades ópticas, tal como o aumento dos valores de "gap" óptico das amostras.
$\mathrm{O}$ resultado de emissão FL mais intenso está associado às amostras de $\mathrm{SrSnO}_{3}$ contendo $1 \%$ em mol de lantânio a $600{ }^{\circ} \mathrm{C}$, atribuído ao aumento da concentração de aditivos e imperfeições decorrentes da presença de lantânio em promoção ao grau de desordem. O sistema com 10\% em mol de lantânio apresenta evidências de recombinação de defeitos no sentido de diminuir o grau de desordem, levando ao decréscimo da emissão fotoluminescente.

\section{AGRADECIMENTOS}

À CAPES, ao CNPq, à FINEP/CT-INFRA pela concessão de bolsa e pelo financiamento de projetos associados. Ao grupo CMDMC e ao Laboratório Multiusuário de Nanotecnologia - CETENE, pelo apoio na caracterizações dos pós.

\section{REFERÊNCIAS}

[1] R. A. Pereira, A. A. Mendes Filho, F. G. D. S. Araújo. Rev. Esc. Minas 59 (2006) 207. 
[2] M. R. Nascimento, M. R. C. Santos, S. J. G. Lima, C. D. Pinheiros, J. W. M. Espinosa, E. Longo, A. G. Souza, I. M. G. Santos, Cerâmica 54, 329 (2008) 120.

[3] Y. Shihimizu, M. Shimabukuro, H. Arai, T. Siyama, J. Electrochem. Soc. 136 (1989) 1206.

[4] S. Wang, M. Lu, G. Zhou, Y. Zhou, A. Zhang, Z. Yang, J. Alloy. Compnd. 432 (2007) 265.

[5] M. C. F. Alves, M. R. Nascimento, S. J. G. Lima, P. S. Pizani, J. W. M. Espinosa, E. Longo, L. E. B. Soledade, A. G. Souza, I. M. G. Santos, Mater. Lett. 63 (2009) 118.

[6] C. P. Udawatte, M. Kakihana, M. Yoshimura, Solid State Ionics 128 (2000) 217.

[7] B. Hadjarab, A. Bouguelia, M. Trari, J. Phys. Chem. Solids 68 (2007) 1491.

[8] D. F. Shiver, P. W. Atkins, Química Inorgânica, $4^{\circ}$ Ed, Bookman, Porto Alegre, RS (2008) 296.

[9] M. M. Vijatovic, B. D. Stojanovic, J. D. Bobic, T. Ramoska, P. Bowen. Ceram. Int. 36 (2010) 1817.

[10] L. G. P. Simoes, M. O. Orlandi, A. L. Araujo, M. R. D. Bomio, E. R. Leite, E. Longo, Cerâmica 49, 312 (2003) 232.

[11] L. G. P. Simões, M. O. Orlandi, P. R. Bueno, E. R. Leite, E. Longo, J. Electroceram. 10 (2003) 63.

[12] L. G. P. Simões, M. R. Cássia-Santos, M. M. Oliveira,
E. Longo, J. A. Varela, Mater. Chem. Phys. 90 (2005) 234. [13] M. Licheron, G. Jouan, E. Husson, J. Eur. Ceram. Soc. 17 (1997) 1453.

[14] G. Pfaff, V. D. Hildenbrand, H. Fuess, J. Mater. Sci. Lett. 17 (1998) 1983.

[15] F. M. Pontes, C. D. Pinheiro, E. Longo, E. R. Leite, S. R. Lazaro, J. A. Varela, P. S Pizani, T. M. Boschi, F. Lanciotti, Mater. Chem. Phys. 78 (2002) 227.

[16] J. H. Rangel, N. L. V. Carreño, E. R. Leite, E. Longo, C. E. M. Campos, F. Lanciotti, P. S. Pizani, J. A. Varela, J. Lumin. 99 (2002) 7.

[17] D. L. Wood, J. Tauc, Phys. Rev. B. 5 (1972) 3144.

[18] W. F. Zhang, J. W. Tang, J. H. Ye, Chem. Phys. Lett. 418 (2006) 174.

[19] L. P. Ravaro, L. V. A. Scalvi, A. S. Tabata, F. M. L. Pontes, J. B. B. Oliveira, J. Appl. Phys. 114 (2013) 084304.

[20] A. T. de Figueiredo, V. M. Longo, R. O. Silva, V. R. Mastelaro, A. Mesquita, R. W. A. Franco, J. A. Varela, E. Longo, Chem. Phys. Lett. 544 (2012) 43.

[21] P. G. Mendes, M. L. Moreira, S. M. Tebcherani, M. O. Orlandi, J. Andrés, M. S. Li, N. Diaz-Mora, J. A. Varela, E. Longo, J. Nanoparticle Res. 14 (2012) 750.

(Rec. 26/06/2013, Rev. 19/12/2013, Ac. 25/01/2014) 